\title{
Efeu (Hedera helix) - mehr als beliebter Bodendecker und Fassadenbegrüner
}

ARMin Jagel \& INGo HetZel

\begin{abstract}
Ivy (Hedera helix) is the only native species of the aralia family. It is widespread as a shrub and climber. There are numerous cultivars, some of which are grown as house plants. The plant is toxic but also serves as a medical plant. Biology, ecology, use and mythology of ivy are summarized. Some relatives of Hedera helix are described.
\end{abstract}

\section{Zusammenfassung}

Efeu (Hedera helix) ist das einzige heimische Araliengewächs. Als Bodendecker und Kletterpflanze ist er in Natur und Garten weit verbreitet. Zudem gibt es viele, als Zimmerpflanzen kultivierte Sorten. Das giftige Gewächs ist außerdem eine traditionelle Heilpflanze. Biologie, Ökologie, Verwendung und Mythologie des Efeus werden vorgestellt. Ergänzend werden einige weitere mit dem Gewöhnlichen Efeu verwandte Arten kurz beschrieben.

\section{Weit bekannt und trotzdem außergewöhnlich}

Der Gewöhnliche Efeu (Hedera helix) ist eine Pflanze der Ausnahmen. Bei uns ist er der einzige heimische Vertreter der Araliengewächse (Araliaceae), die sonst überwiegend in den Tropen vorkommen. Außerdem ist Efeu einer der wenigen einheimischen immergrünen Gehölze, die mit ihren Wurzeln klettern. Auch seine späte Blütezeit, die Überwinterung der unreifen Früchte und die Ausbildung verschiedener Blattformen (Heterophyllie) sind in unserer Flora ungewöhnlich.

\section{Namen und Namensherküinfte}

Der deutsche Name Efeu (engl. ivy) entstammt dem germanischen Kulturkreis und hat sich aus den althochdeutschen Bezeichnungen ebahewi bzw. ebah (ca. 750-1050 n. Chr.) und den mittelhochdeutschen Namen ephhöu bzw. ebehöu (ca. 1050-1250 n. Chr. ) entwickelt (CARL 1995). Der Gattungsname Hedera geht auf die Bezeichnung bei den Römern zurück. Diese wiederum bezieht sich vermutlich auf die griechische Vokabel hedra (= sitzen), wodurch darauf hingewiesen wird, dass die Pflanze auf einer Unterlage festsitzt (DÜLL \& KuTZELNIGG 2005). Der Art-Beiname helix (von griech. helissein = gewunden, spiralig) dagegen ist irreführend, da Efeu anders als z. B. Hopfen (Humulus lupulus) nicht windet.

Weitere im deutschsprachigen Raum heute gebräuchliche Namen sind Eppig, Eppich oder ge- legentlich Pfau als Weiterentwicklung aus der Schreibweise Epheu. Seltener werden die Namen Ewig, Immergrün und Wintergrün benutzt, die sich auf die immergrüne Belaubung beziehen (vgl. MARZELL 1972). Grab-Efeu deutet an, dass $H$. helix äufig als Bodendecker auf Friedhöfen gepflanzt wird. Stellenweise tauchen die Bezeichnungen Baumtod oder Totenranke auf, da den Haftwurzeln eine Schadwirkung auf Bäume nachgesagt wird.

\section{Verbreitung und Lebensraum}

Hedera helix ist eine schattentolerante, ozeanisch verbreitete Art, deren Schwerpunkt in luftfeuchten und wintermilden Regionen Mitteleuropas liegt. Efeu bevorzugt nährstoffreiche Böden mit guter Wasserversorgung, besiedelt aber auch schwach saure Substrate. Sein geschlossenes natürliches Areal erstreckt sich von der Iberischen Halbinsel, Italien und Griechenland nordwärts bis zu den Britischen Inseln und nach SüdostSkandinavien. Die Ostgrenze reicht vom Baltikum über die Karpaten bis zum Schwarzen Meer. Im Südosten der USA gilt Efeu mittlerweile als eingebürgert, nachdem er dort 1750 eingeführt wurde (SCHUCK 2006).

In Mitteleuropa ist $H$. helix eine Charakterart der sommergrünen Laubwälder von der Ebene bis in submontane Höhenlagen. Efeu gilt in Mitteleuropa neben Stechpalme (Ilex aquifolium) und Buchsbaum (Buxus sempervirens) als immergrünes Tertiär-Relikt. Nordost-Spanien, der südliche Alpenrand und der Balkan waren Refugien, 

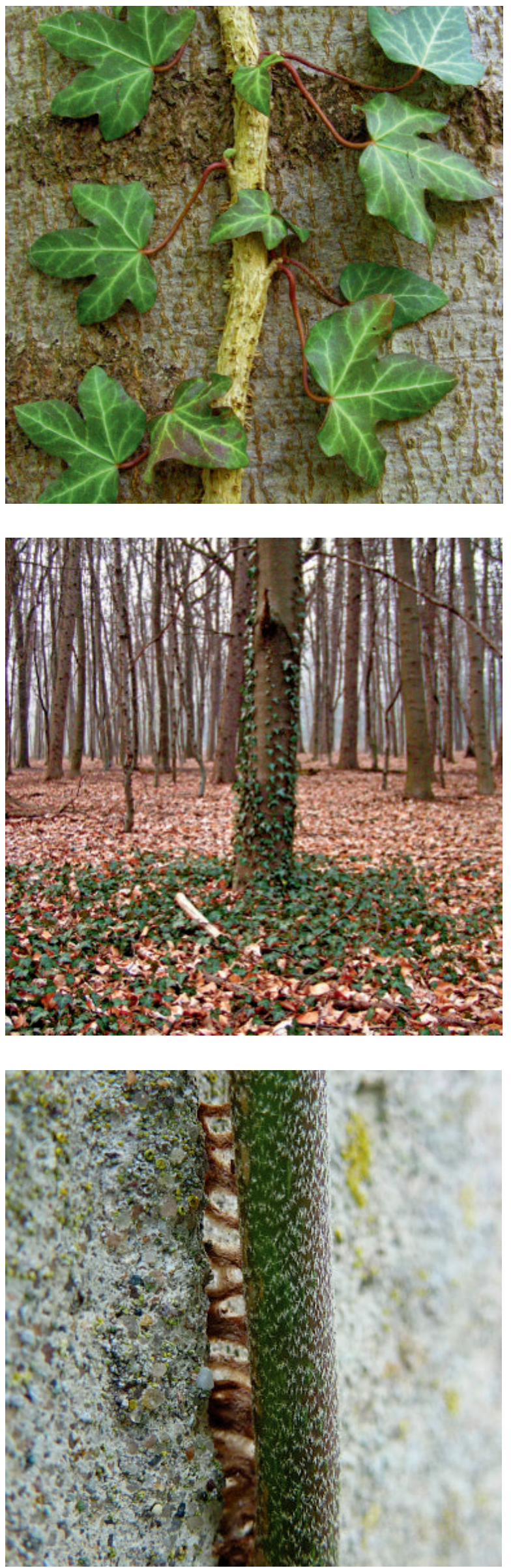

wo Efeu die Eiszeiten überdauern konnte. Am Ende des Boreals, vor etwa 8000 Jahren, erreichte Efeu bereits seine heutige Verbreitung (DierschKe 2005 a). Neuere Studien heben die Bedeutung von Efeu als Bio-Indikator für den Klimawandel in Mitteleuropa hervor. Eine mancherorts deutliche Vergrößerung der Bestände in den letzten Jahren wird als Hinweis für die $\mathrm{Zu}$ nahme milderer Winter gedeutet. Neben der vegetativen Ausbreitung am Boden, bei der Efeu oft durch Schneeauflagen vor Frostschäden geschützt wird, ist vor allem eine vermehrte Ausbildung der Kletter- und Baumform als Hinweis auf eine Klima-Erwärmung zu deuten, denn bei kletterndem Efeu können Fröste stärkere Schäden als bei der Bodenform hervorrufen (vgl. auch WALTHER 2002, DierSCHKE 2005 b).

\section{Morphologie 4.1 Wurzeln, Sprossachse und Blätter}

Efeu bildet sprossbürtige Wurzeln. Vor allem in klimatisch günstigen, wintermilden Gegenden dienen diese als Haftwurzeln, mit denen die Zweige an Bäumen, Mauern und Felsen bis $20 \mathrm{~m}$ hoch klettern können (Abb. 1, 2, 3). Nährstoffe nehmen diese Haftwurzeln nicht auf. Sie entstehen in der Regel auf der lichtabgewandten Seite und werden vor allem an stark beschatteten Sprossen gebildet. Sie entwickeln sich gelegentlich aber auch rings um den Zweig. Efeu kann mehrere hundert Jahren alt werden, wobei dann dickere Stämme einen Umfang von $2 \mathrm{~m}$ erreichen können (FUKAREK 2000).

Efeu wird häufig fälschlicherweise als Schmarotzer bezeichnet, obwohl er mit seinen Wurzeln nicht in andere Pflanzen eindringt, um ihnen Nährstoffe oder Wasser zu entziehen. Die Pflanzen schaden ihren Trägerbäumen allenfalls durch hohes Gewicht oder Beschattung.

Die immergrünen, ledrigen und glänzenden Blätter haben variable Formen, die von tief ge-

Abb. 1 (oben): Kletternder Efeu-Zweig an einem Baumstamm.

Abb. 2 (Mitte): Beginnende Ausbildung der Kletter- und Baumform in einem Wald im Ruhrgebiet.

Abb. 3 (unten): Junger kletternder Efeu-Spross an einer Betonwand. 
lappt bis vollkommen ungelappt reichen (Abb. 6). Blüten bildende Sprosse entwickeln rautenförmige Altersblätter (Abb. 7). Die Ausprägung unterschiedlicher Blattformen heißt Heterophyllie. Wird Efeu vegetativ über Stecklinge aus einer Altersblatt-Region vermehrt, bleibt die Blattform erhalten, was für gärtnerische Züchtungen genutzt wird. Im Winter sind Efeu-Blätter häufig rötlich überlaufen. Da die umgebenden Gehölze nun kahl sind, werden die Efeu-Blätter stärkerer Strahlung ausgesetzt, weshalb als Sonnenschutz rötliche Anthocyane eingelagert werden (Abb. 8). Möglicherweise dient die Substanz aber auch als Frostschutz.

\subsection{Blüten und Früchte}

Blütenstände bilden sich nur an nicht zu stark beschatteten Sprossen. Die Blüten stehen in einfachen, aufrechten Dolden (Abb. 9). Efeu ist das einzige heimische Gehölz, bei dem sich die unscheinbaren, gelblich grünen Blüten erst im Herbst (meist September bis Oktober) öffnen. Sie produzieren reichlich Nektar. Als Bestäuber findet man an den für den Menschen eher unangenehm riechenden Blüten insbesondere Fliegen. Sie sind aber auch für Bienen als Futterquelle attraktiv (HESS 1990, DüLL \& KuTZELNIGg 2005). Erst im Frühjahr reifen die bläulich-schwarzen Beeren heran (Abb. 11,12).

\section{Heilkraft und Giftwirkung}

Efeu wird schon lange als Heilpflanze genutzt. Er wurde deshalb vom Verband Deutscher Drogisten zur Arzneipflanze des Jahres 2010 gewählt. Apotheken bieten heute mehrere Präparate gegen Husten, Bronchitis, Asthma bei Kindern und vor allem gegen Keuchhusten an, die in erster Linie aus Blättern, Blüten und Zweigspitzen gewonnen werden (vgl. PAHLOW 2006). In der Volksmedizin wird ein Tee aus getrockneten Efeu-Blättern beschrieben, der z. B. gegen Kopfschmerzen, Hautkrankheiten, Kopfschuppen oder Rheuma Anwendung fand und z. T. immer

Abb. 4 (oben): Alter, dicker Efeu-Stamm mit großen Haftwurzeln an einer Betonwand.

Abb. 5 (unten): Efeu am Stamm einer Stiel-Eiche (Quercus robur) im Winter im Botanischen Garten Bochum.
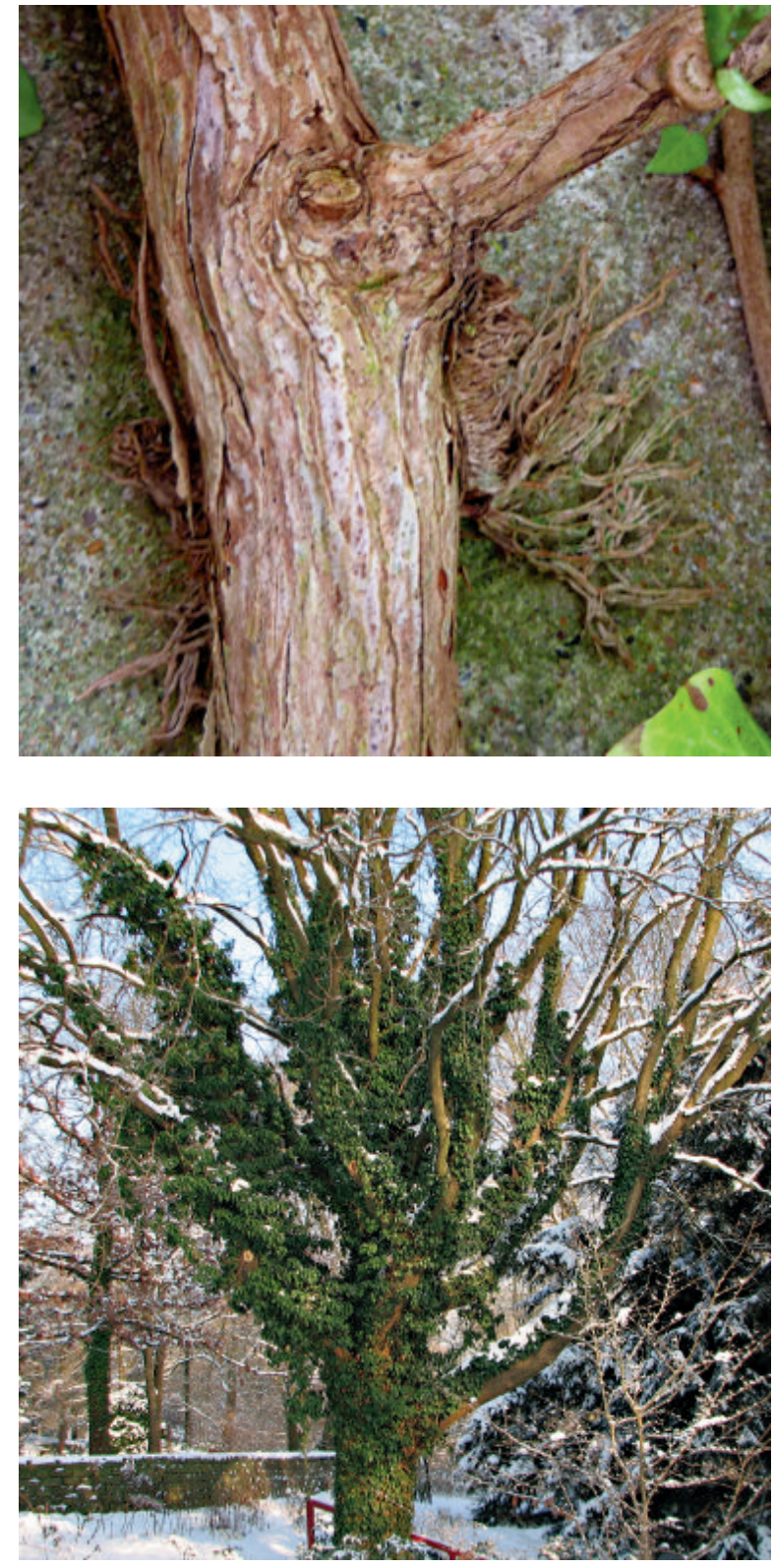

noch findet (PAHLOW 2006, SCHERF 2008). Auch in der Homöopathie wird Efeu schon lange vielseitig verwendet. Durch seinen Jodgehalt wird Efeu vor allem gegen Überfunktionen der Schilddrüse eingesetzt. Zudem wird Efeu gegen Entzündungen der oberen und unteren Atemwege, des Magen-Darm-Traktes, der Leber, Galle und Bauchspeicheldrüse sowie gegen Rheuma und Gicht benutzt (vgl. Hillier \& MelziG 2003). Das Harz der Luftwurzeln diente bis in das 20. Jh. als Grundlage für Zahnfüllungen.

Alle Organe des Efeus sind je nach Dosierung aber auch giftig. Als besonders toxisch gelten die Beeren, wobei aber der unangenehm bittere Geschmack die Aufnahme einer gefährlich hohen 

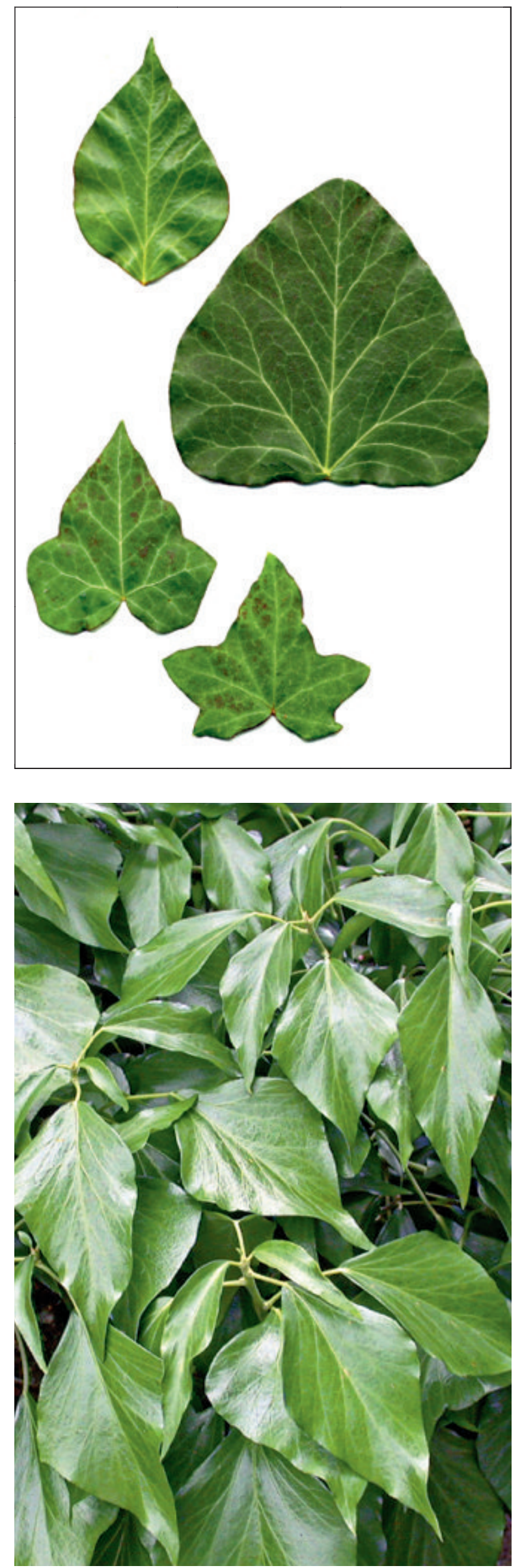

Menge in der Regel verhindert. Früchte und Blätter enthalten eine Reihe verschiedener Inhaltsstoffe wie z. B. Alkaloide und Saponine. Letztere sind vor allem durch Hedera-Saponin C vertreten sowie durch das hochtoxische Abbauprodukt Hederin. Bei Verzehr größerer Mengen können insbesondere bei Kindern schwere, bis zum Tod führende Vergiftungen auftreten. Charakteristische Symptome sind z. B. Übelkeit, Erbrechen, Kopfschmerzen, schneller Puls, scharlachartiger Ausschlag, Benommenheit bis zum Delirium und Atemstillstand (ROTH et al. 2008). Beim Zurückschneiden von Efeu kann es bei empfindlichen Personen zu allergischen Hautreaktionen kommen.

\section{Weitere Nutzungen}

Seit alters her wird Efeu als Zierpflanze verwendet und spielt insbesondere bei der Fassadenbegrünung eine wichtige Rolle. Aufgrund seiner Haftwurzeln braucht er dabei keine Rankhilfe, sofern der Untergrund nicht zu glatt ist. Es wird immer wieder diskutiert, inwiefern die Haftwurzeln den Untergrund durch die Haftwurzeln schädigen oder ihn gar zerstören. Dies dürfte maßgeblich von der Beschaffenheit der bewachsenen Oberfläche abhängen. Efeu schützt durch seine dachziegelartig angeordneten Blätter die bewachsenen Fassaden vor Regen und wirkt als Wärme-Isolierung. Im Gartenbau wird Efeu häufig als Bodendecker gepflanzt, wobei viele verschiedene Sorten mit unterschiedlicher Form und Musterung der Blätter angeboten werden.

Efeu-Blätter wurden früher auch zum schonenden Waschen von dunkelfarbenen Textilien oder zum Dunkelfärben von Bekleidung oder Haaren verwendet. Das harte Holz diente als Ersatz für Buchsbaum bei der Anfertigung von Holzschnitten (SCHERF 2008).

\section{Mythologie und Aberglauben}

Wegen seiner immergrünen Belaubung symbolisiert Efeu Lebenskraft, Freundschaft und eheliche Treue. Die Treue wurde besonders auf Frauen be-

Abb. 6 (oben): Variabilität und Heterophyllie des Gewöhnlichen Efeus, das oberste Blatt ist ein Altersblatt.

Abb. 7 (unten): Altersblätter. 
zogen, denn Efeu galt aufgrund seiner Anlehnungsbedürftigkeit als weiblich (BEUCHERT 2004). Zudem wurde er als göttliches Gewächs angesehen. Schon im alten Ägypten war Efeu Symbolpflanze des Gottes OsIRIS, der Sterben und Wiederauferstehung in der Natur personifizierte. Im antiken Griechenland spielte Efeu im Kult um den Gott des Weines DiONYSOS eine herausragende Rolle. Wie die Weinrebe war er dem Gotte heilig und wurde oft ornamental dargestellt. Der lange Thyrsosstab des Dionysos endete in einem Knauf aus Efeu-Blättern. Efeu und Wein gehörten zusammen, denn damals wurden Weinreben mit Efeu-Zweigen festgebunden.

Griechische Priester überreichten Brautpaaren bei der Vermählung eine Efeu-Ranke als Zeichen ewiger Liebe und dauernden Glücks. Zur Steigerung der ehelichen Treue war Efeu in der Rezeptur einer erotisierenden mittelalterlichen Hexen-Salbe enthalten, die allerdings aufgrund weiterer Zutaten wie Eisenhut (Aconitum spec.) und Wasserschierling (Cicuta virosa) nicht ungefährlich war. Damit Efeu auch seine gewünschte, magische Kraft entfalten könne, sollte er eine Stunde vor Sonnenaufgang und mit nach Osten gewandtem Gesicht geschnitten werden (MALIZIA 2002). Neben seiner Anwendung als Liebespflanze wurde Efeu auch als Verhütungs- und Abtreibungsmittel genutzt und für weitere, nicht weniger zweifelhafte Anwendungen empfohlen. So galt das Binden einer Ranke um den Kopf als sicheres Mittel gegen Trunkenheit (HAERKÖTTER \& HAERKÖTTER 1986). Das Auftragen eines wässrigen Efeu-Extrakts sollte weibliche Fettpölsterchen schrumpfen lassen.

Nach deutschem Volksglauben durfte man Efeu nicht im Hause haben, denn er bringe Unglück, zerstöre den ehelichen Frieden oder bedeute sogar den Tod eines Angehörigen. Als Begründung dafür wurde der bevorzugte Wuchsort des Efeus auf Gräbern und Ruinen angegeben (HAERKÖTTER \& HAERKÖTTER 1986).

Abb. 8 (oben): Ungelapptes Blatt mit winterlicher Rotfärbung durch Einlagerung von Anthocyanen.

Abb. 9 (Mitte): Blütenstand.

Abb. 10 (unten): Auch Schmetterlinge wie hier ein Admiral besuchen Efeu-Blüten.
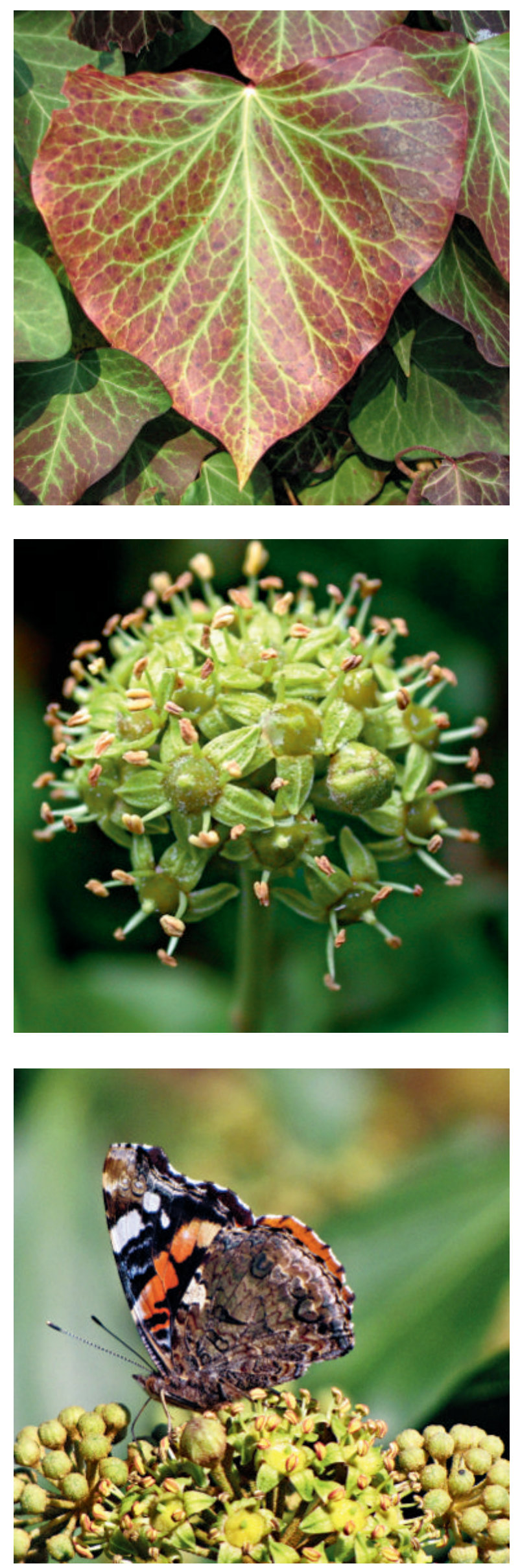

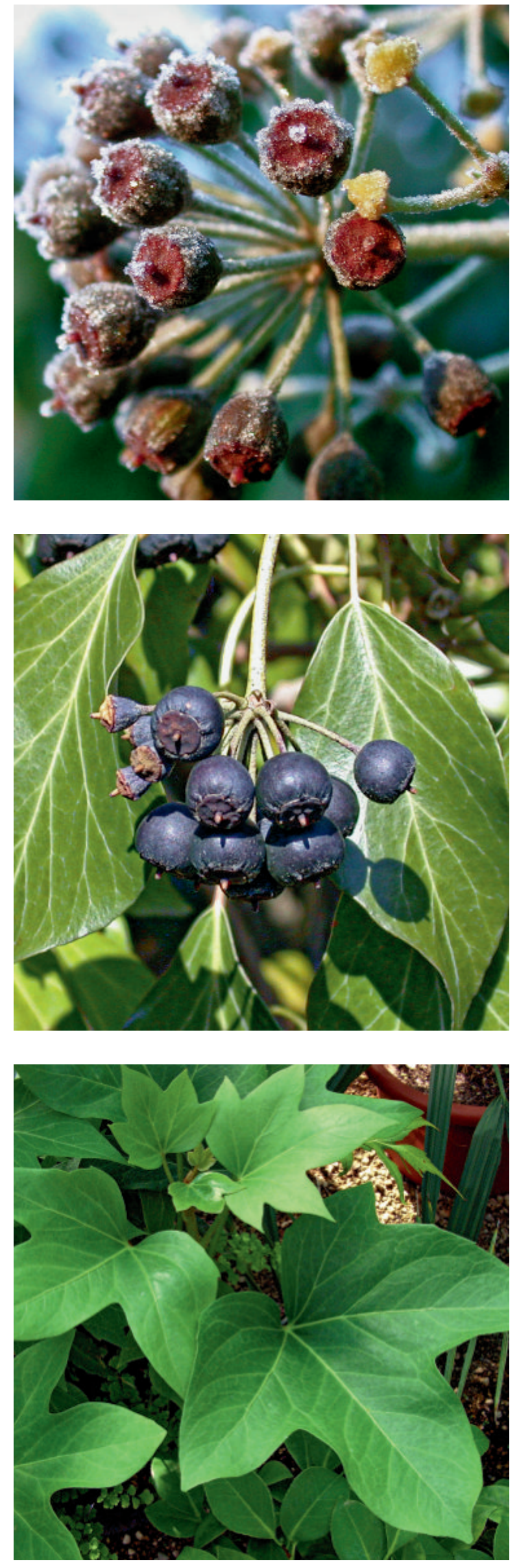

\section{Weitere Arten}

Viele Sorten Efeu-ähnlicher Hängepflanzen für Blumenampeln gehen nicht unbedingt auf Hedera helix zurück. Sie gehören oft zur Verwandtschaft des Kanarischen ( $H$. canariensis agg.) oder des Atlantischen Efeus (H. hibernica). Die Sortenvielfalt ist unübersichtlich und eine Zuordnung zu einer Art oft nicht eindeutig zu treffen. Erschwerend ist dabei, dass die genannten Efeu-Arten manchmal als Unterarten von $H$. helix angesehen werden, oder anderseits der Kanaren-Efeu in mehrere sehr ähnliche Kleinarten (H. canariensis, $H$. algeriensis, $H$. azorica, $H$. maderensis) mit getrennten Arealen aufgeteilt wird. In Gartencentern werden die Sorten meist allgemein als "Hedera helix in Sorten" ausgezeichnet. Zur Blüte gelangt Zimmer-Efeu meist nicht, es sei denn, man erwirbt Sorten, die aus Zweigen mit Altersblättern vermehrt wurden (z. B. Hedera helix 'Arborescens'). Die meisten Zimmer-EfeuPflanzen sollten nicht ins Freie gepflanzt werden, weil sie aufgrund der Herkunft ihrer Ursprungsarten bei uns nicht ausreichend winterhart sind.

Eine weitere beliebte Zimmerpflanze ist die Efeu-Aralie ( $\times$ Fatshedera lizei, Abb. 13), eine Hybride aus der Zimmer-Aralie (Fatsia japonica, Abb. 14)) und dem Atlantischen Efeu (Hedera hibernica, RÜCKER 2005)

Bei uns im Freien kultivierbar ist außer $H$. helix der Kolchische Efeu (Kaukasus-Efeu, H. colchica, Abb. 15, 16)). Er stammt aus SüdostEuropa, Kleinasien und dem Kaukasus. Er wird in Gärten gepflanzt und verwildert mittlerweile auch mancherorts wie z. B. in Wäldern des Ruhrgebietes (FuCHS et al. 2006). Hedera colchica ähnelt dem Gewöhnlichen Efeu, hat aber größere, weniger gelappte Blätter, größere Früchte und höhere Wärmeansprüche (RÖBER et al. 1991). Nach Roloff \& BÄrTELS (2006) zeichnet er sich außerdem durch einen Sellerieduft aus.

Abb. 11 (oben): Unreife, mit Raureif benetzte Früchte im Winter.

Abb. 12 (Mitte): Reife Früchte.

Abb. 13 (unten): Die Zimmerpflanze $\times$ Fatshedera lizei, eine Hybride aus dem Atlantischen Efeu (Hedera hibernica) und der Zimmer-Aralie (Fatsia japonica). 


\section{Literatur}

Beuchert, M. 2004: Symbolik der Pflanzen. - Frankfurt. CARL, H. 1995: Die deutschen Pflanzen- und Tiernamen. Deutung und sprachliche Ordnung. Reprint der 1. Aufl. von 1957. - Wiesbaden.

DiersChKe, H. 2005 a: Zur Lebensweise, Ausbreitung und aktuellen Verbreitung von Hedera helix, einer ungewöhnlichen Pflanze unserer Flora und Vegetation. - Hoppea 66: 187-206.

DIERSCHKE, H. 2005 b: Laurophyllisation - auch eine Erscheinung im nördlichen Mitteleuropa? Zur aktuellen Ausbreitung von Hedera helix in sommergrünen Laubwäldern. - Ber. Reinh.-Tüxen-Ges. 17: 151-168.

DülL, R. \& KuTzelnigg, H. 2005: Taschenlexikon der Pflanzen Deutschlands. Ein botanisch-ökologischer Exkursionsbegleiter. 6. Aufl. - Wiebelsheim.

FuCHS, R., FukAREK, F. 2000 (Hrsg.): Urania-Pflanzenreich. Blütenpflanzen 1. - Berlin.

Haerkötter, G. \& Haerkötter, M. 1986: Hexenfurz und Teufelsdreck. Liebes-, Heil- und Giftkräuter: Hexereien, Rezepte und Geschichten. - Frankfurt.

Hess, D. 1990: Die Blüte. Struktur, Funktion, Ökologie, Evolution. - Stuttgart.

Hiller, K. \& Melzig, M. F. 2003: Lexikon der Arzneipflanzen und Drogen. - Berlin.

Marzell, H. 1972: Wörterbuch der deutschen Pflanzennamen. - Leipzig.

MALiZIA, E. 2002: Liebestrank und Zaubersalbe. Gesammelte Rezepturen aus alten Hexenbüchern. - München. PAHLOW, M. 2006: Das große Buch der Heilpflanzen. 2. Aufl. - Augsburg.

RÖber, R., Roloff, A., BÄrTels, A. 2006: Flora der Gehölze. Bestimmung, Eigenschaften und Verwendung. 2. Aufl. - Stuttgart.

Roth, L., Daunderer, M. \& KormanN, K. 2008: Giftpflanzen, Pflanzengifte. - Hamburg.

RÜCKeR, K. 2005: Die Planzen im Haus. - Stuttgart. SCHERF, G. 2008: Alte Nutzpflanzen wieder entdeckt. München.

SCHUCK, H. J. 2006: Hedera helix LINNÉ, 1753. - In: SCHÜtT, P., WeIsGerber, H., SCHUCK H. J., LANG, U. M., Stimm, B. \& Roloff, A. (Hrsg.): Enzyklopädie der Sträucher. - Hamburg.

WALTHER, G.-R. 2002: Weakening of climatic constraints with global warming and its consequences for evergreen broad-leaved species. - Folia Geobotan. 37: 129-139.

Abb. 14 (oben): Zimmer-Aralie (Fatsia japonica).

Abb. 15 (Mitte): Kolchischer Efeu (Hedera colchica): Blätter an kletternden Zweigen.

Abb. 16 (unten): Kolchischer Efeu (Hedera colchica): Blütenstand.
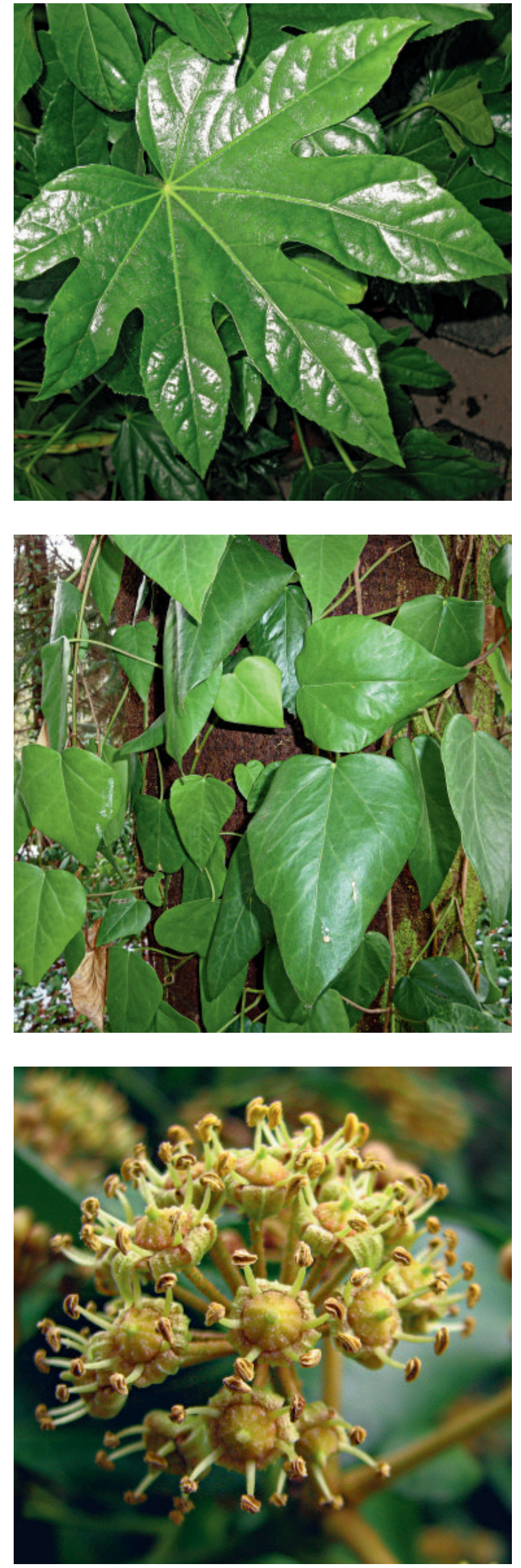